\title{
NARRATOLOGIE MÉDIATIQUE ET MÉDIAGÉNIE DES RÉCITS
}

\author{
Philippe Marion ${ }^{1}$
}

Depuis Platon, bien avant lui sans doute, la notion de récit a suscité nombre de débats théoriques et de polémiques. Que de controverses engendre encore sa seule définition... Comme le récit fait vibrer certaines cordes essentielles de l'identité humaine, il n'y a aucune raison pour que le phénomène s'interrompe un jour.

Il serait naîf de croire que la dimension "médiatique", dans tout ce qu'elle suggère d'exposition et de visibilité, offre l'opportunité de délimiter le territoire du récit et donc de mieux l'appréhender. Car si le médiatique équivaut à une restriction -de la même façon qu'un corpus constitue, pour les analystes de contenu, une réduction de l'univers- il est aussi, dans un mouvement inverse, l'occasion de proliférations et de métamorphoses. Comme l'exprime Ricœur :

De nouvelles formes narratives, que nous ne savons pas encore nommer, sont déjà en train de naître, qui attesteront que la fonction narrative peut se métamorphoser, mais non pas

\footnotetext{
1 Professeur au Département de communication de l'Université catholique de Louvain.
} 
mourir. Car nous n'avons aucune idée de ce que serait une culture où l'on ne saurait plus ce que signifie raconter' ${ }^{1}$.

Malgré quelques affleurements irréfutables, le récit semble gagner en complexité lorsque la fonction narrative se déplace et s'étoile au gré des territoires des médias. Face à cette évidence complexe du récit médiatique, face aussi aux perspectives de recherche que laisse entrevoir la narratologie médiatique, le texte qui suit repose lui aussi sur l'articulation d'un double mouvement.

La première partie, la plus longue, s'inscrira dans une perspective d'ouverture. Pour poser les bases encore fragiles d'une narratologie médiatique, il faut confronter le récit médiatique à quelques traits fondamentaux du médiatique lui-même. Quelles dimensions sont engagées sous cette étiquette, quelles composantes révèle cette appellation dans l'état actuel de notre vie commune avec les médias? Dans un mouvement opposé, la seconde partie opérera un repli sur une seule de ces dimensions évoquées et qui me semble déterminante. Il s'agira d'interroger la narratologie médiatique à partir d'une brève saisie comparative des spécificités et des ressemblances propres à certaines configurations médiatiques courantes. On y abordera les phénomènes de médiagénie et de transmédiagénie.

Ouvrir des questionnements, créer ou exacerber des problématiques. Pour se développer, la narratologie médiatique doit se mesurer à de telles incertitudes fécondes. Dans cet esprit, les propos de cet article feront surtout germer des questions d'objet et de cadre disciplinaire tout en proposant quelques hypothèses de réponse.

\section{Petit incipit narratologique}

Avant de brosser un portrait de la narratologie médiatique, quelques propositions générales s'imposent afin d'intégrer l'analyse des récits dans le contexte de la communication narrative. Car le récit n'est ni un énoncé inerte, ni une "fabula" désincarnée. Il doit aussi être envisagé dans son aptitude communicationnelle, dans la relation que sollicite son énonciation ${ }^{2}$. Si la narratologie porte sur des récits,

1 P. Ricceur, Temps et récit, t. 2, La configuration du temps dans le récit de fiction, Paris, Éd. du Seuil, 1984, p. 42.

2 Voir notamment les travaux de Van Dijk qui insiste sur le processus de narration : «The narrative is seen as being an integral part of the communicative or interactional process. From the pragmatic-linguistic perspective this means that all 
elle se doit surtout d'examiner la narration en tant que passage à l'acte. En tant que pragmatique relationnelle.

\section{Narration historique, narration discursive}

Sur le plan de l'instance d'énonciation tout d'abord, se pose forcément la question des modalités de présence -ou d'absence- du narrateur, problématique à laquelle on associe volontiers le nom de Genette. Il s'agit alors, comme le rappellent Adam et Revaz, de considérer l'instance de narration comme énonciation narrative' ${ }^{1}$.

Dans cette mesure, il semble possible de recadrer, dans le champ spécifique de l'énonciation narrative, le fameux binôme discours/histoire conçu par le linguiste É. Benveniste et rendu célèbre par Barthes. Pour opérer ce recadrement narratif, il faut dépasser une difficulté apparente, celle du terme récit lui-même, souvent utilisé pour désigner la forme histoire ${ }^{2}$. Il me paraît important, cependant, de ne pas cantonner le récit dans la seule forme de l'énonciation de type... récit (histoire) et d'admettre notamment que certaines narrations -et particulièrement certaines formes ambiguës de narrations médiatiques- ont recours à des modes discursifs d'énonciation. C'est souvent le cas lorsque le présentateur d'un journal télévisé nous interpelle pour nous raconter l'actualité. Ou lorsqu'un éditorialiste nous prend à partie, avec force déictiques, pour reconfigurer pour nous (avec nous...) le sens des événements de l'actualité.

A ces deux formes opposées d'énonciation délimitées par Benveniste dans le cadre général de la langue, je propose donc de faire correspondre solidairement deux formes opposées d'énonciation narrative. Avec la narration à tendance discursive, une priorité est donnée à l'opacité de la médiation racontante. A l'autre extrême du spectre énonciatif, une narration à tendance historique laisse s'exprimer toute la transparence du monde raconté en occultant autant que

factors relevant to the process must be taken into consideration». T.A. VAN DIJK, "Narrative Analysis", in Handbook of discourse analysis, vol. 2, London, Academic Press, 1989-1992.

1 J.-M. AdAM et F. REvaz, L'analyse des récits, Paris, Éd. du Seuil, 1996.

2 Pour Benveniste, le discours est «une énonciation supposant un locuteur et un auditeur, et chez le premier, l'intention d'influencer l'autre». Avec la forme histoire qui, selon l'auteur, englobe à la fois le récit de fiction et celui des historiens, le locuteur tente de s'effacer de sa propre parole. Voir É. BENVENISTE, Problèmes de linguistique générale, Paris, Gallimard, 1966, chap. 19. 
faire se peut les traces de narration. Cette organisation binaire est précieuse car elle permet de réaliser une première ventilation des énonciations narratives complexes. Pensons au "syncrétisme" de la monstration et de la narration pratiqué par nos médias audio-scriptovisuels.

\section{Narration et relation}

Lorsque l'on échange de l'information par la voie du récit, on se place dans un cadre, dans un système codé. Un système où se tresse un réseau d'instructions et d'attentes cristallisant le jeu relationnel complexe entre narrateur et narrataire. Les partenaires de la communication narrative acceptent donc d'honorer respectivement les termes d'un contrat ${ }^{1}$ implicite, d'un pacte ou d'un protocole de lecture. Selon Ricœur, c'est

une note de confiance par lequel il est demandé d'accorder au romancier non seulement le droit de savoir ce qu'il raconte ou montre, mais de suggérer une appréciation, une estimation, une évaluation de ses personnages principaux. Droit exorbitant que le lecteur concède à l'auteur' ${ }^{2}$.

Quant au positionnement du récepteur tel que le programme l'énonciateur au sein de son message, le raconteur tente de gérer les "horizons d'attente"3 de celui auquel il s'adresse. De son côté, celui-ci essaie d'interpréter les instructions qu'il croit déceler dans ce qu'il reçoit en produisant des hypothèses interprétatives. C'est tout l'univers de la gestion de l'intrigue, la dynamique de dramatisationapaisement, bref le schème fondamental tension-détente, celui-là même qui sculpte toute existence humaine.

1 Le terme de contrat est assez largement répandu et dépasse actuellement le champ strict de la narratologie, ainsi, par exemple, «le contrat d'information médiatique» défini par P. CHARAUDEAU in Le discours d'information médiatique, Paris, NathanINA, 1997, p. 67 et sv. Certains cependant n'acceptent pas ce terme, car ils le considèrent comme abusif. Ainsi F. Jost suggère de le remplacer par «promesse», cf. les débats tenus au colloque de Cerisy, «Penser la télévision», en juin 1997.

2 P. Riceur, Temps et récit, t. 3, Le temps raconté, Paris, Éd. du Seuil, coll. Points, 1985 , p. 296.

3 Pour cette notion d'horizon d'attente, on peut se référer aux théoriciens de la réception littéraire tels W. ISER, "La fiction en effet", Poêtique, $\mathrm{n}^{\circ} 39$, sept. 1976, p. 279. 
Dans ce sens, raconter devient synonyme de l'art subtil de mélanger -de confondre volontairement- cognition et émotion, affect et information. Selon Mieke Bal, l'activité narrative consiste à suspendre l'étanchéité des catégories de l'information et de l'affect. Dans le cadre du contrat narratif, la rétention et la distribution de l'information deviennent l'objet d'un jeu de dévoilement dont le rythme, la cadence, l'inflexion dramatique vont forger le désir et le plaisir de savoir du récepteur. Ce qui équivaut, dans le cadre narratif, à stimuler son affect : plus il ressent le désir cognitif de découvrir et de comprendre la suite de l'intrigue -autrement dit, plus il s'adonne à ce que Eco appelle la "coopération interprétative"1-, plus il s'investit émotivement dans le récit.

\section{Le double visage de l'illusionné}

En ce qui concerne l'attitude de réception narrative, elle se caractérise par la décision implicite d'accepter les règles du système et de s'y soumettre, c'est-à-dire de jouer le jeu du récit et celui du genre dont on estime qu'il relève. Pour que l'interaction narrative s'épanouisse, il faut que le consommateur de récit entre dans un jeu herméneutique : où veut-on me mener, que veut-on me faire imaginer ou reconstruire du monde, quelle position dois-je prendre pour entrer dans cette partie narrative ? Surtout étudiée dans le cadre de la fiction, cette attitude -cet état spectatoriel, disent les filmologues- $s$ 'appuie sur une position "schizoïdique". Pour entrer en fiction, il faut accepter d'être dupé tout en sachant qu' on l'est. Pour mieux être dedans, il faut qu'une parcelle de nous reste dehors. Octave Mannoni résume cette position paradoxale par la formule "je sais bien, mais quand même". Je sais bien que c'est une histoire, mais quand même j'y crois. "C'est un peu comme si, ajoute-t-il à propos du spectateur de théâtre, nous menions avec nous la personne à mystifier". Il ne faut pas l'oublier, par son étymologie le mot "illusion" signifie grosso modo : "entrée dans le jeu". Une expression comme "illusion ludique" serait dès lors pléonastique ${ }^{2}$.

Roger Odin adopte un point de vue assez proche lorsqu'il commente, dans le cadre du cinéma, le paradoxe de la fictivisation :

1 U. ECo, Lector in fabula, Paris, Grasset, 1985 (1979).

2 O. MANNONI, Clefs pour l'imaginaire ou l'autre scène, Paris, Éd. du Seuil, 1969, p. 165. 
[Le spectateur] sait bien qu'il est au cinéma et qu'on lui raconte une histoire, mais il sait encore que cette histoire ne cherche pas à l'atteindre personnellement ; c'est alors qu'il sera le plus sûrement touché au plus profond de lui-même, et bel et bien en tant que personne réelle'.

De manière comparable, Clément Rosset décrit la structure fondamentale de l'illusion comme étant un art de percevoir juste mais de tomber à côté dans la conséquence :

l'illusionné fait ainsi de l'événement unique qu'il perçoit deux événements qui ne coïncident pas, de telle sorte que la chose qu'il perçoit est mise ailleurs et hors d'état de se confondre avec elle-même. Tout se passe comme si l'événement était magiquement scindé en deux, ou plutôt comme si deux aspects du même événement en venaient à prendre chacun une existence autonome ${ }^{2}$.

Cette attitude paradoxale qui caractérise la participation à la fiction, mériterait, me semble-t-il, d'être généralisée à notre manière globale de "consommer" le récit. D'où cette hypothèse : accepter les termes implicites du pacte narratif, c'est accepter d'entrer dans un jeu, même si le substrat événementiel appartient au monde réel, tel celui que couvre l'actualité journalistique. L'interaction narrative s'appuierait alors sur un "conditionnement" primaire du récepteur. La forme narrative réussie mobiliserait donc une attitude de réception déterminée qui se caractériserait par une complicité interprétative spécifique influençant notre rapport au monde raconté. Dans tous les cas, la narration convoquerait contractuellement une suspension d'incrédulité. Et ce serait seulement l'importance et l'amplitude de celle-ci qui différencierait, sur un plan plus subsidiaire, le récit factuel du récit fictionnel ${ }^{3}$.

A ces suggestions théoriques s'ajoute l'hypothèse d'une configuration imaginaire proprement médiatique qui, à son tour, viendrait déployer et amplifier l'imaginaire propre à l'interaction narrative. L'effet de fiction fondamental inhérent au processus de narration -j'y reviendrai ci-dessous- se trouverait alors à la fois déplacé et

1 R. ODIN, "Du spectateur fictionnalisant au nouveau spectateur", Iris, $\mathrm{n}^{\circ} 8,2 \mathrm{e}$ sem. 1988 , p. 129.

2 C. ROSSET, Le réel et son double, Paris, Gallimard, 1976, p. 16.

3 Cf. A. Gaudreault et Ph. Marion, "Dieu est l'auteur du documentaire", in "Le documentaire", Cinémas, vol. 4, n² 2, Montréal, hiver 1994. 
hypertrophié dans les narrations médiatiques. L'observation de tels mouvements est un des défis majeurs que la narratologie médiatique se doit de relever.

\section{Le médiatique ou le récit sous influence}

Dans la polysémie qui est sienne et malgré les difficultés à la cerner, la seule appellation de médiatique cristallise plusieurs dimensions importantes sur les plans conceptuels, définitionnels et méthodologiques. Ces différentes dimensions affectent et modifient à leur manière les apparences et les dynamiques du récit. Une narratologie médiatique ne peut donc les ignorer. Mais les composantes de notre "nouvelle culture médiatique" sont nombreuses, enchevêtrées et mouvantes. Les traits retenus ci-dessous ne sont donc pas exhaustifs, mais ils constituent des points d'ancrage importants susceptibles de mieux appréhender les enjeux de la communication narrativomédiatique.

\section{Médiatique et quête du court-circuit}

L'expérience médiate est, on le sait, liée à une inévitable distanciation représentationnelle du réel, a fortiori lorsque cette "mise en scène du monde" procède des grands médias qui tendent à déplacer plus ou moins implicitement notre rapport à ce monde. Paradoxe du médiatique : il se sert de la médiateté -l'irréductible fracture propre à toute représentation- pour donner l'illusion d'une possible immédiateté.

L'illusion médiatique d'immédiateté s'est manifestement renforcée au fil de l'histoire des médias. Selon le principe médiologique du "confort médiatique", un bon média travaille à se faire oublier comme si sa transparence était garante de l'impression que le monde "réel" nous parvient sans médiation. Dans cet esprit, le progrès médiatique désigne "tout dispositif permettant d'enfouir le moyen terme (entre le monde et nous), ou de raccourcir le circuit d'accès"1. Pensons aux images virtuelles, où tout l'effort porte sur le raccourcissement même de l'accès, sans se soucier d'une quelconque réalité du référent. 1 D. Bougnoux, Sciences de l'information et de la communication, Paris, Larousse,
coll. "Textes essentiels", 1993, p. 531. 
Une narratologie médiatique ne peut analyser son objet qu'en prenant en considération cette idéologie de la transparence, cette quête du court-circuit. Il est vrai cependant que les conséquences de cette situation dépassent de loin le seul récit médiatique. Selon Augé, il faut constater plus généralement un affaiblissement des rhétoriques et des groupes intermédiaires face à deux processus : l'internationalisation de l'humanité et l'individualisation des consciences. Selon lui, l'impression de maîtriser le temps -et donc l'illusion d'immédiateté très sensible dans le direct télévisé, par exemple- serait technique mais non symbolisée ${ }^{1}$.

Par ailleurs, la médiologie s'est donné comme but de désenfouir cette occultation des médias. Désenfouissement d'autant plus urgent que les omniprésentes métaphores de la caresse et du surf introduisent à la nouvelle société du contact. C'est, selon Debray, l'idyllique trilogie de l'immédiateté-proximité-simplicité :

Nous voilà sommés de préférer le flux à la forme, le jaillissement à l'élaboration, (...) le regard de plongée (participatif, fusionnel) au regard de surplomb (critique, objectivant). Tout alentour réclame de la présence immédiate plutôt que du représentée .

D'où une volonté médiologique de réhabiliter le spectacle dans son sens ancien parce qu'il permet l'empathie tout en maintenant la distanciation. Car dans notre nouvelle culture médiatique, nous manquerions de dramaturgie et donc de distance. Selon certains historiens, tel Gouhier, c'est par la perte du sens dramatique que s'expliqueraient les plus grands drames de l'histoire.

La narratologie médiatique ne pourrait-elle pas, dans le domaine qui est le sien, lutter contre la perte du sens narratif ? Ou, peut-être, lutter contre la perte de conscience d'être en contact quasi permanent avec des récits d'autant plus prégnants que leur nature narrative est occultée ? Si cette hypothèse est fondée, la narratologie médiatique devrait se donner comme tâche de désenfouir les récits médiatiques que nous consommons sans le savoir alors même qu'ils forgent notre identité collective.

1 M. AUGÉ, Le sens des autres, Paris, Fayard, 1994. Voir aussi l'article du même auteur dans ce numéro.

2 R. DEBRAY, "Pourquoi le spectacle?", Les Cahiers de médiologie, n 1, Paris, Gallimard, 1996. 


\section{Factuel/fictionnel : une opposition désactivée ?}

Par ailleurs, les performances de nos médias et la fascination de l'immédiateté tendent à généraliser la fiction. De nombreux auteurs ont montré à quel point factuel et fictionnel perdaient en étanchéité sous la pression contextuelle du médiatique. Certains évoquent même la perte de pertinence et de légitimité de cette opposition. C'est la position d'Augé lorsqu'il développe l'idée du "tout fictionnel":

Cette invasion, c'est celle des images, on l'aura deviné, mais c'est, bien plus largement, le nouveau régime de la fiction qui affecte aujourd'hui la vie sociale, la contamine, la pénètre, au point de nous faire douter d'elle, de sa réalité, de son sens et des catégories (l'identité, l'altérité) qui la constituent et la définissent ${ }^{1}$.

Avec les images virtuelles et les "représentation intégrales", par exemple, la réalité de l'image semble -formule célèbre- avoir pris le dessus sur l'image de la réalité. Sur un plan plus narratif, on sait qu'il suffit d'un passage au médiatique pour qu'une personne réelle se transforme en personnage. Or, évoquer le personnage, c'est convoquer l'univers du récit.

Une solidarité intense unit fiction et narration, par l'intermédiaire de la vectorisation et de la mise en intrigue du temps. Si les médias généralisent la fiction, pourrait-on s'attendre alors à ce qu'ils généralisent solidairement le narratif ? Ou peut-être est-ce parce qu'ils généralisent le narratif que s'instaure ce vaste mouvement de fictionnalisation? Narration/effets de fiction, fiction/effets de récit, la narratologie se doit aussi de réinterroger les liens entre ces termes, dans le contexte médiatique.

\section{La circulation, le flux}

On considère souvent que pour être pleinement médiatique, un récit doit se développer dans plusieurs médias. C'est alors qu'il existe un courant transversal assez fort pour subjuguer les velléités -les réticences- identitaires de chacun de ces médias. Leurs caractéris-

1 M. AugÉ, La guerre des rêves. Exercices d'ethno-fiction, Paris, Éd. du Seuil, 1997, p. 11. 
tiques propres doivent s'accommoder de ces scénarios circulants. Le mot d'ordre est la porosité active, la propagation réciproque.

Dans ce profil, le médiatique désigne des contenus, des idées, des formes, des personnages, etc. qui possèdent une aptitude transmédiatique souvent assortie d'une propension au glissement intergénérique : ils se propagent aisément de la presse écrite au cinéma, de la radio à la télévision, du journalisme à la publicité, du reportage au feuilleton... Ils inspirent la création de sites sur Internet et sont même l'objet de jeux vidéos ou de CD-Roms. Ainsi en est-il, par exemple, du grand récit particulièrement sensible actuellement de la quête de sécurité pour l'enfance. Ou, si l'on adopte un point de vue négatif sur le même sujet, celui des multiples dangers qui menacent aujourd'hui ${ }^{1}$ les enfants.

Ces processus de flux et de circulation, particulièrement actifs dans notre culture médiatique, pourraient être rapprochés mutatis mutandis de la conception picturale et esthétique de "l'optique" développée notamment par Riegl, Wölfflin ou Deleuze. "L'œil optique effleure linéairement alors que l'œil haptique pénètre en profondeur, il s'englue dans les volumes"2. Ce cheminement linéaire, effleurant, ne le retrouve-t-on pas dans ces récits médiatiques qui, comme dépourvus d'aspérités, traversent les médias et les genres en se chassant les uns les autres? La pratique du surfing, si chère aux internautes, est sans doute une des manifestations les plus évidentes de cette conception optique du glissement.

Le flux médiatique demanderait bien sûr un développement plus étayé. De manière simple, on pourrait tout d'abord l'envisager sur un plan diachronique. C'est l'idée d'un chassé-croisé des "contenus", d'une narrativité "horizontale" (syntactique), construite sur la succession de récits ou de fragments de récits. Pensons à l'expression journalistique courante : "une info en chasse une autre", ou encore ce

1 Aujourd'hui? N'est-ce pas aussi parce que l'enfance menacée est devenu un récit particulièrement médiatique que l'impression de menace est à la fois ressentie comme plus actuelle et plus intense. On retrouve en tout cas cette ambiguité lancinante : les médias sont en même temps miroirs (souvent équivoques, selon l'expression célèbre de L. Quéré) et projecteurs.

$2 \mathrm{Ph}$. MARION, Traces en cases, travail graphique, figuration narrative et participation du lecteur, Louvain-la-Neuve, Academia-Bruylant, 1993, p. 176 et sv. J'ai, dans cet esprit, suggéré le concept de «narration optique». 
"robinet d'eau claire"l que semble constituer le modèle du "journal continu" cher à CNN.

Sur un plan synchronique, le médiatique renvoie à la porosité transmédiatique évoquée ci-dessus. Par sédimentations plus ou moins contemporaines, se tressent des narrativités "verticales", construites sur le passage de mêmes contenus dans différents médias et dans différents sites de ces médias.

Le flux diachronique peut être hétérogène ou bien être relié par des liens thématiques, narratifs, symboliques : évolution du récit au fil des événements. Un macro-récit peut aussi se constituer par la combinaison de l'axe synchronique et de l'axe diachronique : jour après jour, des informations se suivent sur le même sujet et circulent dans différents médias. Les affaires socio-politico-judiciaires qui secouent la Belgique et différents pays d'Europe en constituent un bon exemple.

\section{Volatilité et recyclage}

En favorisant ainsi la dynamique du flux, le monde médiatique semble reposer sur une économie paradoxale. Celle-ci n'est pas sans conséquences. D'une part, les contenus médiatiques sont exposés à une usure rapide. On sait, par exemple, à quel point la fraîcheur constitue une valeur clef de l'actualité. Le médiatique se caractérise alors par une sorte de surabondance, de dilapidation, de "gaspillage" de données. Les sujets traités -et surtout montrés- se bousculent dans une fuite en avant. Ils se démodent très vite. Le déjà-vu ne signifie-t-il pas souvent crédibilité affaiblie, du moins dans le contexte d'une information-spectacle? Les campagnes d'intérêt public ou humanitaire sont sans cesse confrontées à ce problème.

Mais, par un mouvement contraire, la culture médiatique contemporaine pratique à grande échelle le recyclage. Des éléments démodés sont revisités, remis au goût du jour ${ }^{2}$ et offerts à une nouvelle consommation. La publicité a fait de cet "art d'accommoder les restes" une spécialité. Que dire de la télévision qui, à coups de

1 L'expression est de A. Woodrow, Information, Manipulation, Paris, Éd. du Félin, 1990, p. 33 et sv.

2 Il ne serait pas inutile de mettre ce trait du médiatique en perspective avec le système de la mode proposé par Barthes. Je pense notamment à ce qu'il nomme l' «épreuve de commutation». R. BARTHES, Le système de la mode, Paris, Éd. du Seuil, 1967, p. 30 et sv. 
"télé-mémoires", se cherche encore confusément une légitimité identitaire?

Reste à savoir si cette transhumance est entretenue par un effet de spirale intrinsèquement médiatique (la traînée de poudre) : chaque média se doit de se réapproprier à sa façon ce qui brille dans la vitrine du voisin. Ou alors ce succès relève-t-il d'une quelconque importance référentielle, tels ces faits exceptionnels qui semblent emballer le système? Ou provient-il encore de la résonance plus ou moins forte qu'il entretient avec des modèles archétypaux ou séculaires, au diapason de la sensibilité et de l'imaginaire collectifs ? Les faits divers les plus médiatiques, par exemple, se déposent sur des architectures narratives éprouvées renvoyant à quelque puissante racine identitaire.

\section{L'autre clôture des récits non clos}

Face à la définition du récit, face à son économie ceinte constituée d'un pendant croissant entre de l'avant et de l'après (le début, la fin ; l'homéostasie initiale, l'homéostasie finale), se pose alors la question décisive de la clôture. Essayons une alternative structurale binaire "à la Bremond". Ou bien le récit médiatique "errant" dans le temps et les médias trouve une forme de clôture sui generis. Ou bien il n'en trouve pas et reste ouvert. A cela s'ajoute une interrogation définitionnelle : un récit non clôturé est-il encore un récit ?

Le glissement de médias en médias pour le même récit constituet-il une clôture ou une ouverture ? La culture médiatique ne favoriserait-elle pas des clôtures paradoxales, des clôtures en spirale. Le récit médiatique devrait alors comprendre sa clôture comme une perpétuelle fuite en avant. Notre identité narrative relève peut-être de l'effet domino. L'impression de clôture pourrait bien résider alors, paradoxalement, dans le maintien (la certitude) d'une perpétuelle ouverture.

Le récit médiatique d'actualité parviendrait-il ainsi à une clôture spécifique ? Dès lors qu'il est aux prises avec des événements inachevés ou à prolongement, ce récit n'a de sens qu'ouvert. Pourtant cette ouverture affecte largement aussi le domaine fictionnel : les séries littéraires, bédéistiques -je pense à la sérialité active des mangas- ou surtout télévisées peuvent apparaître comme interminables, toujours susceptibles de développements et d'arborescences. Comme l'actualité. Comme la vie. Cette non-clôture serait donc compensée par une forme de clôture décalée, déplacée pragmatiquement : la méta-clôture 
rassurante qu'apporte la certitude d'un même qui se prolonge indéfiniment, au fil des séries, par-delà les différences codifiées et apprivoisées des occurrences ponctuelles. Celles-ci peuvent alors, sans dommage quant à elles, demeurer non clôturées.

A côté de ces mécanismes généraux, le système médiatique peut aussi générer des récits explicitement clôturés interrompant le flux. Ainsi en est-il des récits cérémoniels étudiés par Dayan et Katz :

(...) Ces événements jouent un puissant rôle d'intégration. En effet, s'ils sont liés à des conflits, ils parlent avant tout de réconciliation. Presque toujours, ils prônent une cessation des hostilités et c'est ce qui les distingue des nouvelles quotidiennes. (...) Ils correspondent alors aux "figures de guérison" commentées par Sfez (1993). A l'instar de celles-ci, ils "se manifestent avec éclat dans 1'histoire institutionnelle et sociale, mais ponctuellement et peu souvent". Ils jouent un rôle fondateur. Ils ne le jouent qu'un moment ${ }^{1}$.

Ces récits cérémoniaux sont donc bien clôturés, isolés dans le temps et les programmes qu'ils ont la légitimité d'interrompre. Ils constituent des sortes d'îlots dans le flux médiatique. Mais s'ils sont îlots, il n'en restent pas moins très médiatiques au moins pour deux raisons : ils se développent synchroniquement dans différents médias et ils mobilisent un vaste public. Sur le plan narratif, la suspension des hostilités et la résolution des conflits qu'ils manifestent renvoient à l'accès à un nouvel équilibre; ils offrent ainsi à un déséquilibre symbolique et social l'opportunité d'une clôture.

\section{Réseau et vraisemblable médiatique}

Ce qui précède nous amène à ce constat : flux et porosité circulatoires n'amènent pas forcément à une dissémination anarchique. Rhétorique de la propagation, dérive organisée... S'il exacerbe un processus d'étoilement, le médiatique est aussi capable de gérer -spontanément, accidentellement, implicitement, consciemment...ces signifiés migrateurs, objets centraux de la lecture plurielle définie par Barthes. Une narratologie médiatique se doit donc de comprendre le pluriel effectif du texte médiatique dans lequel naissent et croissent les récits médiatiques. Et ce d'autant plus que ce texte médiatique est

\footnotetext{
1 D. DAYAN et E. KATZ, La télévision cérémonielle, Paris, PUF, 1997, p. 10.
} 
particulièrement sujet à ce que Barthes, encore, appelle le "fading des voix" qui hypertrophie le pluriel : "plus l'origine de l'énonciation est irrepérable, plus le texte est pluriel"'.

Le médiatique, lorsqu'il prend la forme d'une dissémination organisée, peut alors se concevoir comme une mise en réseau. Certains "systèmes" fictionnels permettent sans doute de mieux en appréhender les conséquences. C'est le cas du cycle des Cités obscures élaboré par Schuiten et Peeters.

Il s'agit d'un macro-récit protéiforme et polymédiatique. La matrice narrative initiale multiplie les arborescences et est elle-même affectée par les développements qu'elle a inspirés. Un grand nombre de médias sont sollicités : de la bande dessinée au site sur Internet. A la faveur des changements de médias, de nouvelles fictions s'inventent, renouvelant l'esprit de la série. A chaque nouvelle manifestation, le récepteur s'interroge moins sur les nouvelles péripéties que le récit va lui proposer que sur la manière dont le monde des cités va se trouver enrichi et solidairement infléchi.

La consistance progressive de l'univers fictionnel... est fondée sur le flux, la propagation programmatique. Par sédimentation du récit, par autonomisation de certains éléments, par accumulation de références et surtout d'autoréférences, le réseau -tout en croissantgagne en cohérence interne et étoffe par là sa crédibilité et son vraisemblable.

Le monde composé (diégétisé) de Schuiten et Peeters ne me paraît pas éloigné du monde que tissent les grands récits médiatiques factuels lorsqu'ils tressent en réseau des fragments composites. Une fois encore, le récit des "affaires" belges répond à cette volonté de placer dans la cohérence d'un réseau narratif des faits dont les liens ne sont pas nécessairement vrais et démontrés mais dont l'inscription dans le "réseau" est vraisemblable. Et, solidairement, des faits que l'intégration dans le réseau rend vraisemblables... Par là, le "réseau" trouve une opportunité de croissance tout en renforçant sa cohérence.

Il faudrait dès lors s'interroger sur ce qui définit et alimente le vraisemblable médiatique. La cohérence interne offerte par la mise en réseau -surtout lorsqu'elle relaye et démultiplie la mise en intriguene relègue-t-elle pas au second plan une quelconque adéquation à la "réalitê"? L'important se situant dans le réalisme intrinsèque qui émane de la cohésion de la mise en réseau. Les sédimentations

1 R. BARTHES, $S / Z$, Paris, Éd. du Seuil, 1976, p. 63. 
successives des récits médiatiques ne construisent-elles pas une réalité de second degré -un "réel médiatique"- plus homogène, plus vraisemblable, plus maîtrisable que la réalité "de premier degré" ?1.

Il faudra aussi, bien sûr, s'interroger un jour sur les conséquences narratives qu'entraînent les multiples cristallisations technologiques de cette figure du réseau. Le numérique, par exemple, permet la manipulation de l'image à perte de vue, on lui ajoute autant de couches qu'on le désire. Ou encore : le récit peut-il garder indemne sa définition lorsqu'il rencontre les arborescences interactives du multimédia ? Le consommateur peut ainsi devenir personnage de son propre parcours médiatique : en surfant, en s'immergeant dans un jeu vidéo interactif, en se promenant virtuellement dans un musée, il se construit une temporalité individualisée. Il devient gestionnaire et narrateur de son propre récit en train de se construire par lui et avec lui. Ces récits en construction interactive raccourcissent en quelque sorte la boucle mimétique élaborée par Ricœur et posent, une fois encore, de nouvelles questions à la narratologie médiatique.

\section{Le médiatique : de l'exposition à la crédibilité}

On sait que le médiatique renvoie d'abord à "tout ce qui est relatif aux médias"2, et plus spécifiquement aux grands médias de masse de notre époque. Ainsi pour être ou devenir médiatique, un récit ne doit-il pas simplement être médiatisé ? C'est-à-dire se laisser mettre "en représentation" par une instance de production qui va se charger de le transmettre massivement au public.

Outre la confusion qui se manifeste ici entre les supports (le média télévision, l'affiche), les institutions (le média presse, la publicité), ou les activités de médiation (le journalisme, par exemple), l'étiquette médiatique recèle bien d'autres ambiguïtés. Ainsi, elle obéit autant à un principe d'évaluation qu'à une logique de description. Dans le sens commun, le médiatique ne fait pas que renvoyer aux messages rendus accessibles au plus grand nombre et divulgués

1 La culture médiatique pourrait bien alors être caractérisée par une hypertrophie de la mimèsis II de P. Ricœur, la reconfiguration du temps se dilatant démesurément par le potentiel illimité des mises en réseau.

2 Ce point synthétise et prolonge les réflexions que j'ai proposées dans "Le récit médiatique comme modèle d'interprétation", in G. DERĖze (dir.), Tribunes de presse. Études sur la construction journalistique du sport, Louvain-la-Neuve, Academia-Bruylant, 1996. 
par la médiatisation, il comporte une évaluation plus ou moins implicite. Est médiatique ce qui mérite d'occuper les espaces de représentation aménagés par les médias. Dès lors, le médiatique s'empreint d'une coloration d'acquiescement, de succès, de réussite ; il se caractérise par une certaine fortune au plan de la réception. Le Parlement belge, très peu médiatique jusqu'ici (comme tous les parlements) a trouvé une nouvelle notoriété à l'occasion de la retransmission télévisée de la commission d'enquête sur les disparitions d'enfants.

Dans une vision plus interactivement englobante, le label médiatique peut être considéré comme la résultante apparente d'un subtil et précaire équilibre de l'offre et de la demande, ou encore comme une co-construction évaluative où interagissent, dans un contexte "historique" donné, énonciateurs et énonciataires. Une telle interaction ne signifie nullement équilibre des forces, on sait quelle puissance disproportionnée possède en réalité l'offre et les forces de production socio-économiques qui exercent leur pression en amont de l'offre. Cela ne signifie pas non plus entente euphorique. On peut à cet égard épingler les cas de réajustement spectaculaire où le potentiel médiatique présupposé d'une émission ne trouve pas, quand bien même il est annoncé avec grand fracas, sa confirmation du côté de la réception. Certains "grands feuilletons de l'été" ont connu des échecs retentissants...

\section{Le médiatique comme intuition d'une convivialité}

Sur un plan général de réception, plusieurs auteurs ont signalé ce paradoxe des grands médias : ils stimulent l'appréhension individualiste des messages tout en offrant une perspective groupale virtuelle. Il s'agit, selon Wolton, "d'une solidarité diaphane qui s'instaure entre des individus (...) créant une communication sans doute un peu étrange, mais probablement typique de notre société «individualiste de masse»" médiatique ce qui offre la possibilité de combiner exposition individuelle au message et conscience simultanée d'une intégration dans la communauté.

1 D. Wolton, Éloge du grand public, Paris, Flammarion, 1993, p. 130. 
Avec la télévision, par exemple, l'impression de médiatique semble liée, pour le spectateur, à une sorte de conscience groupale non exprimée. Il peut être seul face à l'écran, mais il sait confusément que d'autres, ailleurs, sont là aussi à regarder. "Pour être spectateur, écrit Daniel Dayan, il faut toujours chausser les lunettes d'un public"l. Recevoir une émission, c'est se constituer convive. Soit cette version médiatique du voyageur solitaire : arrivé dans un pays qu'il ne connaît pas, il ouvre la télévision de sa chambre d'hôtel afin de (d'avoir le sentiment de) s'immiscer dans une communauté qui lui est étrangère... Le spectateur ressent alors la proximité de "l'autre absent" et retrouve une collectivité implicite. Avec sans doute aussi l'impression confuse que cette invisibilité n'est que provisoire ou, plus exactement, qu'elle se passe de vérification concrète. Cette étrange convivialité médiatique pourrait, elle aussi, être rapprochée de la manière dont une société reçoit ses récits et se reconnaît en eux. La troisième mimèsis de Ricœur, celle de la refiguration du temps propre aux narrataires, ne suggère-t-elle pas, elle aussi, une convivialité identitaire implicite ? Dans ce cas, la convivialité virtuelle liée à la consommation d'un média comme la télévision pourrait bien interagir avec celle qui relève de l'identité narrative. Ce serait là, en un mot, notre identité médiatico-narrative.

Pouvant aller jusqu'au "passage à l'acte" qui l'ancre dans la réalité, cette consensualité est donc susceptible de déborder sur le vécu des gens : manifestations de soutien, d'émotion, d'indignation, acclamation d'une vedette ou d'un héros, etc. Ainsi, l'événement, lorsqu'il est empreint de passion et qu'il engage un aspect identitaire, permet de bien mesurer la réduction d'écart entre le spectacle médiatisé et le spectacle contemplé, entre l'émotion individuelle et l'émotion collective. L'événement médiatique est riche d'une "praxis" virtuelle, où l'émotion vécue individuellement par l'exposition aux médias peut trouver une résolution en se dissolvant dans celle du groupe.

\section{De la médiagénie à la transmédiagénie}

Les développements de la première partie ont brossé un portrait du "biotope" où évoluent les récits médiatiques. Un portrait très

1 D. DAYAN, "Les mystères de la réception", Le Débat, n 71, 1992, p. 145. 
ouvert s'appuyant notamment sur des critères syntactiques (le flux, la circulation, le réseau), sémantiques et symboliques (la fictionnalisation, la notoriété) ou encore "pragmatiques". En gardant à l'esprit les faisceaux de détermination très prégnants que constituent ces caractères de notre culture médiatique, il s'agit, pour suivre, d'opérer un retour sur les médias eux-mêmes. Car si la narratologie médiatique doit intégrer dans sa réflexion ces déterminismes contextuels, elle ne peut pas ignorer qu'un récit médiatique est aussi un récit qui "rencontre" un média et inversement.

Or un média, nous le savons au moins depuis Mac Luhan, est loin d'être transparent, quand bien même les performances de transmission et le confort référentiel qu'il nous apporte voudraient nous amener à l'impression contraire. "Les médias (note Bougnoux à la suite de Debray) ne se contentent pas d'acheminer, ils sont bourrés d'instructions et de hiérarchies (de jugements de valeur) incorporées". Les médias génèrent leur propres chimères et fictionnent autant qu'ils fonctionnent. Un titre d'émission comme Les enfants de la télé n'est pas simplement métaphorique... Toute forme de représentation implique une négociation, voire un corps à corps, avec la force de résistance propre au système médiatique choisi. Dans quelle mesure, par exemple, un reportage télévisé construit-il le monde autrement qu'un reportage radiophonique, qu'un reportage photographique, voire même qu'un reportage dessiné traitant le même sujet de référence. Quels sont les registres de l'imaginaire que le média peut intrinsèquement stimuler à partir des virtualités propres de sa configuration expressive, de son dispositif et de son usage social ?

\section{Un média n'est pas l'autre}

Chaque média posséderait donc un "imaginaire" spécifique, sorte d'empreinte génétique qui influencerait plus ou moins les récits qu'il rencontre ou qu'il féconde.

Avant d'examiner les composantes qui définissent cette personnalité de nos médias, il convient de se remémorer les domaines hétéroclites que l'on range sous leur bannière. Dans l'acception laxiste des médiologues, ils recouvrent tout ce qui, de la route à l'école en passant par le théâtre, fait circuler les idées en les co-construisant. Ils désignent pêle-mêle des types de discours institutionnalisés (la publicité, la presse...), des moyens d'expression et des matériaux 
sémiologiques plus ou moins combinés (peinture, caricature, chanson...), des supports technologiques et des moyens de transmission (livre, photographie, vidéo, CD-Rom, affiche...), des médias dans le sens plus restrictif des "grands" médias (télévision, radio, presse écrite, bande dessinée, multimédia...). A cela s'ajoutent certains amalgames ambigus entre médias et genres : hebdomadaire d'information, série, talk show... Tous ces champs catégoriels sont loin d'être mutuellement exclusifs et ils s'entrecroisent librement.

De mon point de vue, la narratologie médiatique doit opérer un premier choix et se concentrer en priorité sur les "grands médias". Selon Veron, chacun de ceux-ci se définit comme

un ensemble constitué par une technologie plus les pratiques sociales de production et d'appropriation de cette technologie, lorsqu'il y a accès public (quelles que soient les conditions de cet accès, qui est généralement payant) aux messages ${ }^{1}$.

Il convient donc d'appréhender au mieux le potentiel spécifique d'un média. Ce potentiel est régi notamment par les possibilités techniques du support, par les configurations sémiotiques internes qu'il sollicite et par les dispositifs communicationnels et relationnels qu'il est capable de mettre en place. Le tout se plaçant sous l'influence réciproque d'usages sociaux réels et en constantes évolutions. Spécificité ne signifie nullement séparation ou isolation : pour apprécier ce différentiel médiatique, il faut saisir solidairement les ressemblances et les divergences qu'un média entretient avec ses congénères.

\section{Quelques singularités de la médiativité}

Appréhender la singularité différentielle d'un média, c'est tenter d'en saisir la "médiativite"2. Celle-ci rassemble tous les paramètres qui définissent le potentiel expressif et communicationnel développé par le média. La médiativité est donc cette capacité propre de représenter -et de placer cette représentation dans une dynamique communicationnelle- qu'un média possède quasi ontologiquement.

\footnotetext{
1 E. VERON, "De l'image sémiologique aux discursivités", op.cit., p. 51.

2 Pour l'usage initial de ce néologisme calqué sur la narrativité, voir Ph. MARION, op. cit., p. 278.
} 
Par définition. Même si les discursivités sociales peuvent influencer cette définition et la faire évoluer.

De manière plus systématique, une médiativité peut être appréciée selon la combinaison de quelques dimensions importantes.

Tout d'abord, deux paramètres de nature externe et globale. Il s'agit, d'une part, des conditions de diffusion et de circulation attachées au média observé ; par exemple, le type de périodicité de la presse écrite ou le type de distribution d'un dépliant publicitaire. Il s'agit d'autre part des modalités de "consommation" publique de ce média saisies dans leur interdépendance avec le contexte culturel et social. A ses débuts, la photographie était ainsi considérée comme un substitut des portraits picturaux en vogue chez les notables. Sur le plan du contenu, elle en mimait d'ailleurs certains... clichés tels que la scénographie des postures et la théâtralité des attitudes. En leur temps hérö̈que, la radio ou la télévision constituaient des foyers rares autour desquels des membres de la collectivité se rassemblaient pour partager la réception d'émissions choisies.

A l'autre extrême et sur un plan interne, une médiativité se juge bien sûr en fonction des matériaux sémiotiques d'expression mobilisés par le média. On a depuis longtemps mis en perspective les mécanismes de cognition et d'affect associés respectivement au "digital" et à "l'analogique". De la même manière, on a souvent insisté sur la différence entre les médias "iconiques" et les médias "scripturaux". On tente de même d'évaluer les effets pragmatiques complexes de l'audio-scripto-visuel. Sans parler des orientations imaginaires divergentes que sollicitent des systèmes d'expression différents au sein d'une même catégorie de représentation comme la monstration, par exemple. Entre une caricature signée et une photo d'agence du même personnage médiatique à une époque donnée, que de différences...

La nature du support et les dispositifs techniques qui lui sont associés orientent eux aussi le sens pragmatique d'une médiativité. Songeons à des innovations techniques telles que le système Barco qui permet notamment de conférer une nouvelle dimension "projective" à l'image télévisée pouvant transformer la réception et même agir sur le sens du message. Dans le domaine de la presse, le format du journal ou du magazine est beaucoup moins anecdotique qu'on ne l'imagine souvent lorsqu' on focalise son attention sur le seul contenu informatif véhiculé. Ainsi, l'adoption d'un format tabloïd n'est pas une simple évolution technique, elle interagit à sa manière avec l'information transmise. Ce format peut même contribuer au 
développement d'un genre particulier de récit d'information comme l'attestent les célèbres "tabloïds" anglais.

Une fois encore, il convient d'insister ici sur le fait que les faisceaux de sens ouverts par ces aspects de la médiativité ne sont envisageables que dans leur combinaison et leur interaction. A l'occasion d'une recherche consacrée aux reportages de Paris Match $^{1}$, j'ai eu l'occasion de montrer combien la dynamique du conglomérat scripto-photographique prend son sens lorsqu'on observe son interférence avec l'espace paginal. Lorsqu'il procède à ses "grands" reportages d'actualité, le magazine laisse souvent les photos s'épancher sur toute la surface de la double page. Ces photos n'ont généralement pas d'encadrement apparent et leur frontière se confond avec celle de la page elle-même. Ce dispositif inspire une interprétation pragmatique simple. En saturant ainsi l'espace paginal, la monstration -le simulacre analogique de la réalité- impose sa prégnance. L'événement surpris par l'objectif ne peut être encadré tant il s'impose dans la démesure de son urgence. Comme l'urgence non maîtrisable d'un vivant toujours en expansion. Sans encadrement et par des photos qui se confondent avec le format de la page, le montré tend à effacer, par saturation, les contraintes spatiales du média. Quant au spectateur, il est -par programmation (stratégie) médiatico-discursive- "happé" par la diégèse. Le monde, déployé dans toute son amplitude, se donne à sa contemplation. Ce plein déploiement du monde dans l'espace paginal nourrit une métaphore implicite : ces photos sont le reflet transposé, différé sans doute, mais analogiquement fidèle de l'événement. Entre le support et l'événement s'établit une sorte d'équivalence, de ressemblance pondérée dans et par son adaptation aux contraintes de la médiativitée .

\section{Média homochrone, média hétérochrone}

On le constate aisément, la médiativité concerne directement les orientations pragmatiques que sollicite la "personnalité" -la part d'opacité- d'un média dans son travail de communication. Chaque média induirait ainsi des parcours de lecture, des modes de participa-

\footnotetext{
$1 \mathrm{Ph}$. MARIon, "Propositions pour une médiatique narrative appliquée, lecture d'un reportage photographique de Paris Match", in J. BAETENS et A. GonZALEZ, Le roman photo, Amsterdam - Atlanta, Rodopi, 1996.

2 Ibid., p. 165.
} 
tion et d'interaction se concrétisant en intelligence avec les messages qu'il véhicule. Parmi les facteurs qui déterminent ces modes d'appréhension préformatés, il en est un qui me paraît trop souvent ignoré malgré son importance : la marque temporelle du lien relationnel qui se noue entre l'émission et la réception de "sessions" médiatiques. C'est dans cet esprit que je propose une typologie regroupant les médias selon deux principes simples : homochronie/ hétérochronie.

Dans un contexte hétérochrone, le temps de réception n'est pas programmé par le média, il ne fait pas partie de sa stratégie énonciative. Le "livre", la presse écrite, l'affiche publicitaire, la photographie, la bande dessinée : autant de lieux d'hétérochronie. C'est dire que le temps de consommation du message n'est pas médiatiquement intégré, il ne fait pas partie du temps d'émission. Dissociée du média, cette temporalité d'émission n'a pas la prétention d'entraîner dans son sillage le temps de réception. Soit cette imagerie révélatrice d'un lecteur semblant transporté, les yeux au ciel, par une phrase du récit qu'il a décidé de quitter provisoirement. Un lecteur -ou un spectateur d'image fixe-possède la liberté médiatique de gérer ce provisoire et de rythmer ses allers-retours au texte. Certes, on peut regarder un JT puis se prendre à réfléchir à un sujet déjà développé mais, ce faisant, on contrarie la prosodie homochronique du flux.

En hétérochronie, le destinataire est donc tenu d'imaginer et de gérer la durée de sa réception du message. Gérer la durée du message, c'est le construire à sa façon, combler des vides. Mais en comblant ces vides médiatiques, on se projette, on co-construit le message par une sorte d'absorption singulière.

Un média homochrone se caractérise par le fait qu'il incorpore le temps de la réception dans l'énonciation de ses messages. Ces derniers sont conçus pour être consommés dans une durée intrinsèquement programmée. S'il veut recevoir normalement (contractuellement) ces messages, le destinataire doit ajuster son temps vécu de réception à celui de l'énonciation médiatique. La communication médiatique incorpore alors une symbiose programmée (forcée) entre les partenaires. Art du temps par excellence, la musique offre sans doute la meilleure représentation de cette homochronie. La musique -et a fortiori plus encore la musique médiatisée- n'est rien sans durée fusionnelle. Une durée rassemblant solidairement, ou plutôt confondant émission et réception. Un arrêt sur musique est ontologiquement impertinent. Le son ne peut que s'annihiler ou s'autodétruire, ou au mieux s'évanouir non sans irradier des parcours 
imaginaires en amont (le souvenir) et en aval (l'attente/ le désir). Ce n'est pas le cas de l'arrêt sur image qu'a répandu l'usage du magnétoscope. Le photogramme garde une certaine consistance sémantique, mais il subvertit néanmoins le flux constitutif du récit.

Dans un site homochrone, le temps de réception est absorbé, intégré, dirigé par l'énonciation médiatique elle-même. Apprécier, recevoir le message, c'est en accepter les directives d'émission temporelle. C'est bien sûr ce qui donne une certaine légitimité à cette différenciation académique qui répartit en deux camps bien séparés les spécialistes de l'image fixe et ceux de l'image animée.

Le spectacle cinématographique, les émissions de télévision ou de radio, sont prévus pour une adéquation énonciative de la durée. Cette contrainte syntagmatique n'est pas sans conséquence. S'il prend quelque distance avec la durée programmée par le média, le récepteur prend le risque de rompre la coopération interprétative temporelle programmée par le média homochrone. La pratique du zapping peut se lire alors comme une volonté d'échapper ou plutôt de compenser par des sauts paradigmatiques déviants (du moins, du point de vue intéressé d'une chaîne) cette contrainte syntagmatique de l'homochronie. Dans le cas d'un lecteur, cette rupture ne prête pas à conséquence puisque le temps de réception est dissocié du média hétérochrone. D'où les problèmes de transposition et de passages transmédiatiques.

\section{Narrativité médiatique}

Dans un sens ancien, le narratif désigne ce qui fait le récit. Je propose donc d'employer le terme de "narrativité" non seulement pour désigner un constat (la narrativité "actualisée") mais aussi dans une acception pragmatique de virtualité : on peut constater le caractère narratif de tel objet observé (par exemple, un film de fiction), mais on peut aussi saisir du ferment narratif dans tel autre objet (par exemple, une photographie qui suggère un récit qu'elle ne contient pas). Il faudrait donc différencier le narratif, comme état explicite et affirmé, du narratif comme dimension possible au vu d'une certaine configuration de l'objet observé (qu'il soit un signe, un message, mais aussi, plus fondamentalement, un média). La narrativité serait non seulement un résultat mais elle contiendrait aussi une 
dimension promissive, celle d'un potentiel ou d'un développement virtuel suggérés par la présence simultanée de certains indices.

Recentrée sur les caractéristiques différentielles des médias, la narratologie médiatique doit se pencher sur la rencontre d'un récit et d'une médiativité. Considérée de manière intrinsèque, la narrativité procède et se nourrit de la médiativité. Certains aspects de celle-ci et surtout leur combinaison peuvent en effet présenter des facultés (pré)narratives plus ou moins affirmées.

En quelque sorte, la narrativité intrinsèque utilise sélectivement et à son profit le potentiel de la médiativité. Ainsi ce caractère prénarratif que possède le film par sa seule capacité d'articuler images et photogrammes en un déploiement temporel. Selon le rythme de leur périodicité, les journaux écrits, parlés ou télévisés suggèrent des systèmes d'agencement et de dévoilement de l'information qui souvent recèlent de la narrativité. Une capacité que manifeste le quasi rituel : "affaire à suivre !". Dans les magazines de presse ou dans les affiches publicitaires, une narrativité intrinsèque peut même émaner de la programmation d'un cheminement de lecture entre la photo et le texte. Entre l'image et l'écrit se joue une construction herméneutique, un fragment de mise en intrigue. Les éléments montrés par l'image sont relayés par le texte qui développe à son tour des mécanismes de dramatisation destinés à être eux-mêmes réinjectés dans l'interprétation de l'image. Ce parcours visuel constitue en soi une aventure $d u$ regard qui dédouble et renforce le récit diégétique.

En outre, la narrativité intrinsèque change considérablement de nature et $\mathrm{d}$ 'aspect selon la distribution homochronie/hétérochronie. On connaît la frustration de certains lecteurs de bande dessinée quand ils découvrent les adaptations de leur série en dessins animés ou en film. Le mouvement des personnages n'est plus à imaginer, à construire comme c'est le cas dans le cadre de l'image forcément fixe du média mais il devient, pour ainsi dire, un prêt-à-porter temporel "imposé" par le temps réel de l'énonciation médiatique.

Mais pour que s'actualise la narrativité complexe d'un récit médiatique, il faut que narrativité interne et externe se rencontrent et s'interfécondent. Cette narrativité externe renvoie, on le sait, aux contenus référentiels "importés" par la représentation de l'événement factuel. "Scénario" issu d'un substrat événementiel réel ou imaginé. Dans le cadre du récit de presse, certains événements semblent eux aussi posséder une prédisposition narrative plus ou moins forte. C'est le cas des accidents, des agressions, bref, de tout ce factuel qui rompt 
un équilibre et bouleverse une homéostasie "normale". La "diégétique"1 événementielle obéit souvent à une négentropie propre au méfait.

Forme particulière de la narratologie médiatique, la médiatique narrative $e^{2}$ porte dès lors sur l'analyse de l'intersection dynamique de deux narrativités. Elle part du média pour retrouver les récits qui -pour accéder à la communication- les utilisent. Et inversement. Rappelons que toute "fabula" se doit de prendre corps dans une cristallisation sémiotique, et par extension, dans une cristallisation médiatique. Pour la narratologie médiatique un texte narratif est interactivement un texte médiatique.

\section{La médiagénie et ses résonances}

On connaît la photogénie, la télégénie. Pourquoi, dès lors, ce terme de médiagénie ? A l'origine, il provient d'une réflexion sur les transpositions et adaptations de récits fictionnels. Pourquoi certains récits se montrent-ils si rétifs à l'adaptation ? Pourquoi semblent-ils s'accrocher ainsi à leur site d'origine ? Comment filmer, sans en trahir l'esprit et la teneur, l'incipit de la Recherche du temps perdu: "Longtemps, je me suis couché de bonne heure"?

La médiagénie est un néologisme qu'il m'a semblé pertinent d'introduire dans le cadre de la médiatique narrative. Toute forme de représentation implique une négociation avec la force d'inertie propre au système d'expression choisi. Cette opacité du matériau expressif constitue une contrainte pour que s'épanouisse la transparence relative de la représentation. Il en va de même pour les narrations médiatiques : le récit s'épanouit au diapason de l'interaction de la médiativité et de la narrativité. Mais il est des rencontres plus intenses que d'autres. Chaque projet narratif peut donc être considéré dans sa médiagénie. Les récits les plus médiagéniques semblent en effet avoir

1 Lire à ce sujet les développements proposés par B. GREVISSE dans Le temps des journalistes, Louvain-la-Neuve, ClACO, coll. de la Faculté des sciences économiques, sociales et politiques, 1997.

2 Le projet théorique de la médiatique narrative a déjà été exposé à plusieurs occasions. Voir par exemple, Ph. MARION, "Scénario de bande dessinée. La différence par le média", in "Le scénario de film", Etudes littéraires, vol. 26, n², Québec, Université Laval, automne 1993. Ou encore, A. GAUDREAULT, Ph. MARION, Transécriture et médiatique narrative, communication au Colloque de Cerisy, 1993. 
la possibilité de se réaliser de manière optimale en choisissant le partenaire médiatique qui leur convient le mieux et en négociant intensément leur "mise en intrigue" avec tous les dispositifs internes à ce média. Un film comme Bleu de Kieslowski ne trouve sa justesse expressive que dans et par le cinéma. Conçues pour et au cœur de la dynamique graphique de la bande dessinée, Les Aventures de Tintin sont pratiquement impossibles à adapter sans d'irrémédiables altérations. Ou encore, n'est-ce pas par médiagénie que la saga de la famille princière de Monaco s'épanouit si bien dans Paris Match -ou dans d'autres magazines d'un type similaire-alors qu'elle ne parvient guère à prendre pied en télévision.

La médiagénie est donc l'évaluation d'une "amplitude" : celle de la réaction manifestant la fusion plus ou moins réussie d'une narration avec sa médiatisation, et ce dans le contexte -interagissant lui aussides horizons d'attente d'un genre donné. Évaluer la médiagénie d'un récit, c'est donc tenter d'observer et d'appréhender la dynamique d'une interfécondation.

Plus un récit est médiagénique, moins sa fabula (son "scénario") est autonome; se creuse alors une distance importante entre cette fabula et le récit médiatisé qui la révèle. La médiagénie est pragmatique, c'est une relation, une interaction, non pas un contenu. En outre, elle n'a de sens que dans une actualisation et un contexte historique donné.

\section{Récits médiatiques et transmédiagénie}

Selon cette conception initiale, la médiagénie semble mieux adaptée à des récits uniques (telle histoire particulière ou telle série ou cycle d'aventures). Remarque importante : l'évaluation médiagénique doit être pondérée non seulement en fonction du caractère narratif ou non de l'objet, mais aussi en fonction du degré de généralité de celuici. Car une intrigue particulière, unique, tend "spontanément" à trouver sa force expressive dans l'interaction avec un site médiatique choisi. Il n'en est pas forcément de même, par exemple, pour la médiagénie d'un genre donné. De par sa généralité, un genre tend a priori à être plus mobile et à s'étendre dans plusieurs médias. Songeons au fantastique, au policier ou à la polémique. La nonmobilité définie ci-dessus comme une des caractéristiques d'une forte médiagénie doit donc être reconsidérée en fonction du degré de 
généralité et d'abstraction de l'objet médiatique. Bref, il reste à établir les repaires épistémologiques susceptibles de fixer les niveaux de pertinence de la lecture médiagénique.

On peut néanmoins, dans un premier temps, considérer la médiagénie en tant que principe d'évaluation global s'appliquant sur différents récits médiatiques singuliers. Selon l'intensité de l'interaction d'un média et d'un récit, la médiagénie peut varier entre deux amplitudes extrêmes: elle peut être forte ou faible. Une médiagénie forte repose sur une sorte de coup de foudre : un récit et son média.

Dans un deuxième temps cependant, la prise en considération des récits de presse ou de certains récits fictionnels circulant dans notre culture médiatique rend nécessaire un aménagement de la lecture médiagénique. Il s'agit alors de prendre en considération un récit selon les médiagénies de ses différents traitements médiatiques. Un grand récit journalistique peut ainsi présenter différentes "facettes" susceptibles de se répartir médiagéniquement sur différents sites médiatiques. Le traitement médiagénique désigne alors la manière dont un média a pu rencontrer la facette de ce grand récit qui lui convenait le mieux. Et inversement.

Si l'on prend en considération certains caractères importants de la culture médiatique évoqués plus haut, on peut même considérer que les récits qui y circulent répondent davantage à une logique de glissement et d'étoilement plurimédiatique. Pour analyser les récits médiatiques contemporains et surtout les grands récits de presse, il semble donc utile d'introduire la notion de transmédiagénie. A l'inverse de la médiagénie, celle-ci reposerait sur l'appréciation de la capacité d'étoilement, de circulation, de propagation transmédiatique que possède un récit. Ainsi en est-il du grand récit d'actualité de l'enfance maltraitée.

Cette transmédiagénie d'un récit n'empêche pas d'en observer les sédimentations ou les réappropriations médiagéniques singulières.

Transmédiagénie et médiagénie, déploiement et fermeture : cet article dans son mouvement même, repose sur ce paradoxe annoncé. Mais il est en cela le reflet du parcours réflexif encore tâtonnant de la jeune narratologie médiatique. Le reflet aussi des multiples visages de son objet polymorphe : le récit médiatique. 